\title{
Hereditary Neuropathy with Liability to Pressure Palsy: A Case Diagnosed with a Quick Multiplex Ligation-dependent Probe Amplification Test
}

\author{
(D) Seda Kanmaz1, (1) Erdem Şimşek1, (1) Hepsen Mine Serin1, (1) Murat Kadri Erdoğan², (1) Sanem Yılmaz1, \\ (1) Gül Aktan11, (1) Hasan Tekgül1', (1) Sarenur Gökben¹
}

${ }^{1}$ Ege University Faculty of Medicine, Department of Pediatrics, Division of Child Neurology, İzmir, Turkey

2University of Health Sciences, Tepecik Training and Research Hospital, Clinic of Medical Genetics, İzmir, Turkey

\begin{abstract}
Hereditary neuropathy with a liability to pressure palsies (HNPP) represented by recurrent focal pressure neuropathies is rare in childhood. Here we present a 10-year-old girl admitted to our hospital with a recurrent weakness in her foot and diagnosed as HNPP with a quick Multiplex Ligation-dependent Probe Amplification test revealing PMP22 deletion.
\end{abstract}

Keywords: Hereditary neuropathy, pressure neuropathy, mjultiplex ligation-dependent probe amplification

\section{Introduction}

Hereditary neuropathy with a liability to pressure palsy (HNPP) is known as an autosomal dominant inherited neuropathy (1). It presents with recurrent sensory and motor nerve palsies usually caused by compression or minor trauma. Although HNPP is rarely reported in childhood, it is probably under-diagnosed due to its wide spectrum of clinical manifestations. Early diagnosis is important to provide appropriate genetic counseling to families, to provide appropriate care for these patients, and to prevent unnecessary investigations (2).

HNPP is diagnosed by genetic tests revealing $90 \%$ of cases, including the PMP22 gene, of a $1.5 \mathrm{Mb}$ chromosome 17p11.2 deletion. However, duplications involving the same gene cause a distinct genetic condition, namely CMT1A, which is the most common type representing approximately
70 to $80 \%$ of all CMTs. HNPP also results from PMP22 gene mutations that alter a single amino acid in the PMP22 protein or that lead to the production of an abnormally small protein.

The incidence of CMT1A and HNPP is as high as 1 in every 2.500 persons (3). Electrophysiological studies are important for differential diagnosis to verify the presence of focal abnormalities in HNPP and to guide genetic studies by revealing an underlying demyelinating polyneuropathy (4).

Real-time quantitative polymerase chain reaction is also very sensitive for identifying the PMP22 gene copy number in CMT1A duplication and HNPP deletion (5). The deletion is usually detected by fluorescence in situ hybridisation (FISH). However, this approach is time-consuming and cannot detect small intragenic rearrangements. On the other hand, 
whole-exome sequencing (WES) is not recommended as the first step since the costs are still high and the sensitivity of this technology is not yet high enough (6).

Recently, Multiplex Ligation-dependent Probe Amplification (MLPA) assays have been proposed as a fast, simple and cost-effective technique for the molecular diagnosis of CMT1A and HNPP (7). Here we report a case with HNPP diagnosed with a quick MLPA test.

\section{Case Report}

A 10-year-old girl was admitted with a sudden onset of weakness that started on her left foot fifteen days previously. There was no pain and symptoms were unresponsive to oral methylprednisolone treatment started in the regional hospital with a diagnosis of polyneuropathy one week previously. Her past medical history was not significant with respect to trauma, toxic exposure, injection or infection. However, one year previously she had a similar symptom in her right foot which developed after sitting on her right leg that resolved itself spontaneously within a week. At that time, she was evaluated at another hospital and her cranial magnetic resonance imaging (MRI) was normal. Her family history was unremarkable.

Neurological examination revealed a loss of dorsiflexion ability in her left foot. Steppage gait was present. Bilateral patellar and achilles reflexes were hypoactive. Complete blood count, biochemistry, lipid profile, acute phase reactants, vitamin $B_{12}, E, A$ levels, laboratory tests for vasculitic disorders were normal. Cranial and spinal MRI were also normal.

Electromyography (EMG) revealed electrophysiological findings of bilateral carpal tunnel syndrome, cubital tunnel syndrome and fibular nerve neuropathy. With this history, neurological examination and EMG findings, HNPP was considered as a preliminary diagnosis.

For definite diagnosis, deletion and duplication studies were performed with 9 (nine) probes specific for 5 (five) exons of the PMP22 gene located in the 17 p12 region, TEXT3 (exons 3 and 9) and COX10 (exon 7) genes located in the close vicinity of PMP22 by MLPA method. Heterozygous deletions including all exons of PMP22 plus TEKT3 (exons 3 and 9) and COX10 (exon 7) genes were detected (Figure 1). DNA microarray analysis performed to detect deletion borders revealed a deletion of $1.360 \mathrm{Mb}$ including HS3ST3B1, PMP22 and TEKT3 genes in the 17p12 region.

An ankle-foot orthosis was applied to alleviate right foot drop. Full recovery was observed within one month. Protective pads for elbows or knees were recommended to prevent pressure and trauma to local nerves. The patient was advised to avoid sitting with her legs crossed, leaning on elbows for long periods, repetitive movement of the wrists and rapid weight loss. Written informed consent was obtained from the family for the publication of this case report.

\section{Discussion}

HNPP is characterized by recurring focal pressure neuropathies such as peroneal palsy with foot drop and carpal tunnel syndrome. HNPP is underdiagnosed due to phenotypic heterogeneity. Family history should be carefully reviewed to identify undiagnosed potential HNPP cases (2). The presented case did not have a family history of HNPP and was diagnosed with a quick MLPA test after the initial electrophysiological evaluation.

The clinical spectrum of HNPP ranges from mononeuropathies to recurrent episodes of brachial

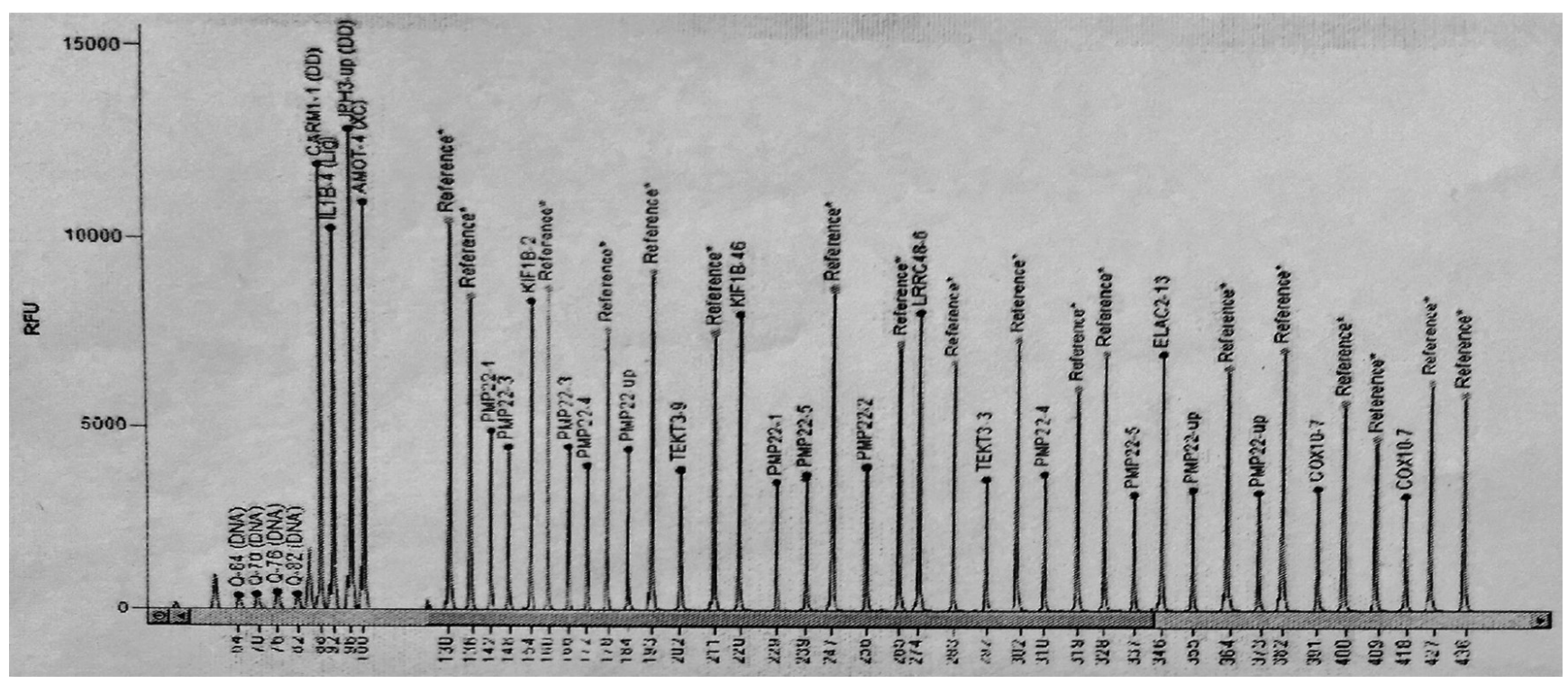

Figure 1. Heterozygous deletions including all exons of PMP22, plus TEKT3 and COX10 
plexopathy. Reportedly, peroneal palsy is the most common presentation (42\%) followed by brachial plexus palsy (2). The most common findings related with HNPP are the existence of polyneuropathy, median terminal motor latency prolongation and multiple compression neuropathies. In our case, there was a weakness only in the left foot while having electrophysiological findings of bilateral carpal tunnel syndrome, cubital tunnel syndrome and fibular nerve neuropathy.

The clinical suspicion of HNPP should be referred to genetic testing, even when study results of nerve conduction do not meet HNPP criteria. Light microscopic changes in nerve biopsy are not specific for this disease. Genetic testing is the first choice because it is noninvasive. PMP22 is the only gene that has been shown to be associated with HNPP. An adjacent gene deletion of chromosome 17p11.2 containing PMP22 is found in about $85 \%$ of the affected individuals, while the remaining $25 \%$ have a pathogenic variant of PMP22 (7).

Recently, a number of tools have been developed for the detection of copy number variations based on new generation sequencing data. WES was recommended in patients diagnosed with CMT1A or HNPP using STR markers to assess the ability of WES to improve the clinical diagnosis. However, use of these methods is limited (4). Due to a large number of genes that can be analyzed by a single technique, the MLPA test represents the gold standard for the molecular analysis of all pathologies derived from the presence of gene copy number variation.

Slater et al. (3) investigated the utility of the MLPA assay in the detection of PMP22 duplications and deletions for the molecular diagnosis of CMT1A and HNPP. The performance of MLPA is compared to one of the interphase FISH analyzes. MLPA assays represent a robust, simple and cost-effective approach for the molecular diagnosis of CMT1A and HNPP (3). The presented case provides additional support to the utility of MLPA assays in the rapid detection of PMP22 duplications and deletions for the molecular diagnosis of patients with HNPP.

In conclusion, HNPP should be considered in recurrent, episodic, painless and entrapment neuropathies after exposure to pressure or trauma. As a result of early diagnosis, high-cost, invasive tests and unnecessary treatments for the prognosis of the disease can be avoided.

\section{Ethics}

Informed Consent: Written informed consent was obtained from the family for the publication of this case report.

Peer-review: Externally peer-reviewed.

\section{Authorship Contributions}

Surgical and Medical Practices: S.K., E.Ş., H.M.S., M.K.E., S.Y., G.A., H.T., S.G., Concept: S.K., H.T., Design: S.K., E.Ş., H.M.S., H.T., Data Collection or Processing: S.K., E.Ş., H.M.S., M.K.E., Analysis or Interpretation: H.M.S., S.Y., H.T., Literature Search: S.K., Writing: S.K.

Conflict of Interest: No conflict of interest was declared by the authors.

Financial Disclosure: The authors declared that this study received no financial support.

\section{References}

1. Bayrak AO, Bayrak IK, Battaloglu E, Ozes B, Yildiz O, Onar MK. Ultrasonographic findings in hereditary neuropathy with liability to pressure palsies. Neurol Res 2015;37:106-11.

2. Chrestian N, McMillan H, Poulin C, Campbell C, Vajsar J. Hereditary neuropathy with liability to pressure palsies in childhood: Case series and literature update. Neuromuscul Disord 2015;25:693-8.

3. Slater $H$, Bruno $D$, Ren $H$, et al. Improved testing for CMT1A and HNPP using multiplex ligation-dependent probe amplification (MLPA) with rapid DNA preparations: Comparison with the interphase FISH method. Hum Mutat 2004;24:164-71.

4. Takahashi S, Chum M, Kimpinski K. Electrodiagnostic characterization of hereditary neuropathy with liability to pressure palsies. I Clin Neuromuscul Dis 2017;18:119-24.

5. Thiel $C T$, Kraus $C$, Rauch A, Ekici $A B$, Rautenstrauss B, Reis $A$. A new quantitative PCR multiplex assay for rapid analysis of chromosome 17p11.2-12 duplications and deletions leading to HMSN/HNPP. Eur I Hum Genet 2003;11:170-8.

6. Jo HY, Park MH, Woo HM, et al. Application of whole-exome sequencing for detecting copy number variants in CMT1A/HNPP. Clin Genet 2016;90:177-81.

7. Beydoun SR, Cho J. Hereditary neuropathy with liability to pressure palsy: two cases of difficult diagnosis. I Clin Neuromuscul Dis 2013;15:28-33. 